\title{
Wovon wir als Lehrperson überzeugt sind und wie wir Gefangene als Lernende sehen
}

\author{
MONIKA SCHUMACHER \\ Zurich University of Applied Science
}

\begin{abstract}
Dieser Artikel bietet einen Einblick in einen Deutschkurs für Fremdsprachige in einer geschlossenen Justizvollzugsanstalt in der Deutschschweiz. Aus der Perspektive der Lehrenden denkt die Autorin über Annahmen und Überzeugungen nach, die man in ein solches, doch eher spezielles Lernsetting mitbringen könnte. Ihren Überlegungen stellt sie Begebenheiten aus ihrem Unterrichtsalltag im Vollzug gegenüber. Sie kommt zum Schluss, dass es nicht in erster Linie darum gehen kann, Überzeugungen einer Lehrperson zu verändern bzw. überwinden - vielmehr ist es im genannten Kontext wichtig, an die Gefangenen und ihr Potenzial als Lernende zu glauben.
\end{abstract}

\section{Key words: Deutsch lehren als Fremdsprache; Überzeugungen; Aspekte der Lehre}

„Jetzt sind Sie also drinnen, wie fühlt sich das an?“ Sie blickte aus dem Fenster seines grosszügigen, hellen Büros: Eine blühende Magerwiese mit Schmetterlingen, ein Idyll, so schien es fast, wären da nicht die blanken blauen Gitterstäbe an den Fenstern gewesen. Diese gaben ihr das Gefühl, in einem engen Raum mit einer sehr niedrigen Decke eingesperrt zu sein...

\section{Einleitung}

Im Collins Cobuild English Dictionary steht zu „belief": „, feeling of certainty that something exists, is true, or is good" ( ein Gefühl oder eine Überzeugung, dass etwas existiert, wahr oder gut ist). Wenn wir unterrichten, tragen wir all unsere Überzeugungen zu den Lernenden, den Methoden, der Unterrichtsorganisation, zum Fach oder Stoff, zu Leistungsmessungen - zu jedem Aspekt, der in irgendeiner Form in das Unterrichten hineinspielt - mit in den Unterrichtsraum (z.B. Pajares 1992). Unsere Annahmen als Fachpersonen sind von unseren eigenen Erfahrungen sowohl als Lehrende als auch als Lernende geprägt. Wir handelt entsprechend unseren Überzeugungen und mit zunehmender Berufspraxis und-erfahrung meist unbewusst.

Im Zusammenhang mit Schule und Bildung sind die Ergebnisse der so genannten Hattie Studie (2009) zu den wichtigsten Faktoren fürs Lernen auf grosses Interesse gestossen und sein Name bzw. derjenige der Studie war in aller Munde. Die Metastudie - über 800 Studien wurden untersucht - brachte an den Tag, dass letztlich nicht etwa die Lerngruppengrösse oder die technische
Ausrüstung in einem Klassenzimmer entscheidend für den Erfolg des Unterrichts sind. Nein, es ist die Lehrperson, die als wichtigster Faktor ausgemacht wurde. Nun könnten wir erleichtert feststellen, dass wir auch in Zeiten, wo immer mehr Technik in den Unterricht Einzug hält, nicht arbeitslos werden. Gleichzeitig sollten wir im Zusammenhang mit den Erkenntnissen der Studie auch bedenken, dass unsere Rolle als Lehrerin oder Lehrer eine enorme Verantwortung mit sich bringt.

Die Ergebnisse der Studie unterstreichen, was bereits frühere Untersuchungen in einem kleineren Rahmen gezeigt haben: Die Lehrperson hat einen grossen Anteil an der Leistung bzw. am Erfolg der Lernenden (z.B. Puchta 1999:257) und am individuellen Lernprozess. Es liegt deshalb nahe, zu untersuchen, welche Überzeugungen die Basis unseres Unterrichts bilden (Yero 2001/2,2).

Die folgenden Überlegungen wurden im Zusammenhang mit dem Deutschunterricht für Fremdsprachige in einer geschlossenen Justizvollzugsanstalt für männliche Gefangene in der Deutschschweiz angestellt. Für diesen Unterricht gibt es keinen Lehrplan, vielmehr kann die Lehrende ihre eigenen Ziele setzten. Die Lernenden können auf ein Sprachdiplom vorbereitet werden - wir kommen später darauf zurück, aber abgesehen davon kann der Unterricht frei gestaltet werden. Das mag befremdlich klingen in einer Institution, die so stark durchstrukturiert ist und eine so hohe Regeldichte hat wie eine Institution des Freiheitsentzugs. 
Diese Freiheiten in der Unterrichtsgestaltung, die grösser sind als in anderen Unterrichtskontexten, geben der Lehrkraft natürlich eine gewisse Macht über die Lernenden. Wie sind die bewussten und weniger bewussten Überzeugungen der Lehrerin im Zusammenhang mit dieser besonderen Gruppe von Lernenden?

In einem Gefängnis zu unterrichten, kann man nicht lernen. Auch wenn man über einen ordentlichen Erfahrungsrucksack verfügt, ist es eine Herausforderung. So hat es auch die Autorin des Artikels erlebt: Als sie im geschlossenen Vollzug zu unterrichten begann, hatte sie bereits einige Unterrichtserfahrung. Sie gab Kurse für Studierende mit einem meist akademischen Hintergrund, hatte Sprachkurse für die IT-Leute einer Bank gegeben und in einer öffentlichen Schule in einem städtischen Quartier mit um die 80 \% Fremdsprachige unterrichtet.

Die Schüler in der Anstalt sind von der Welt draussen abgeschnitten, sie leben in einer isolierten Welt - diese Tatsache spielt auch in den Unterricht mit hinein, und zwar in unterschiedlicher und manchmal völlig überraschender Weise. Man könnte nun sagen, dass der Unterricht in einem Gefängnis sehr eingeschränkt ist, weil man etwa verschiedene heute selbstverständliche technische Möglichkeiten nicht nutzen kann. Das könnte einerseits an den in der jeweiligen Institution fehlenden finanziellen Mitteln liegen, andererseits an zu beachtenden Sicherheitsvorschriften - man kann die Gefangenen nicht einfach so im Internet surfen lassen -, dazu kommt ein aktuell eher restriktives politisches Klima im Land. So würde es die Öffentlichkeit nicht gutheissen, wenn die Gefangenen für ihre Fehltritte statt bestraft auch noch „,belohnt" würden. Nun ja, offensichtlich sind die Überlegungen, was den Schülern im Vollzug für Möglichkeiten entgehen, nur begrenzt sinnvoll, denn Letztere tragen, so Hattie, nicht wesentlich zum Lernerfolg bei.

In der folgenden Diskussion werden fünf Aspekte des Unterrichtens im Vollzug genannt, über die im Zusammenhang mit Ansichten und Überzeugungen reflektiert wird. Es sind dies:

1. Gefangene als Sprachschüler

2. Unterrichtsführung und Methodik

3. Der Einsatz und Sinn der Muttersprache im Unterricht

4. Ansätze und Zugänge

5. Themen

\section{Die Ausgangslage}

\subsection{Die zu unterrichtende Sprache: Deutsch als Fre- mdsprache (DaF)}

In der Schweiz ist die deutsche Hochsprache zunächst die geschriebene Sprache; ihr zugrunde liegt eine Grammatik und Orthographie, die beide streng standardisiert sind. Hochdeutsch ist aber auch die gesprochene Sprache in den Schulen, den elektronischen Medien - wie Fernsehen und Radio - ebenso in offiziellen Situationen. Wenn die Sprache mündlich verwendet wird, ist sie je nach Sprecher oder Sprecherin mehr oder weniger stark dialektal gefärbt. Lokale und regionale Dialekte sind üblich in der alltäglichen mündlichen Kommunikation und fliessen auch in die geschriebene Sprache ein, so wird sie gern von der Jugend verwendet, wenn diese per i-Phone mit Gleichaltrigen kommuniziert.

Fremdsprachige im Justizvollzug der Deutschschweiz sind mit dem hochdeutschen Standard und mit den Schweizer Dialekten konfrontiert. In machen Anstalten können die Gefangenen auf freiwilliger Basis Deutsch lernen, um sich den institutionellen Alltag zu erleichtern. Die Vollzugsmitarbeitenden wiederum können die Gefangenen unterstützen, indem sie Hochdeutsch sprechen und dabei konsistent sind. So kann auch die Kommunikation an der institutionellen Basis effizienter gestaltet werden.

\subsection{Die Vollzugsanstalt}

Die Anstalt Pöschwies in Regensdorf bei Zürich ist die grösste geschlossene Vollzugsanstalt in der Schweiz. Nach Schweizer Gesetzgebung sind die Gefangenen zur Arbeit verpflichtet. Gleichzeitig haben sie, wie etwa in der genannten Anstalt die Möglichkeit, eine Berufslehre in einem der 19 Gewerbebetriebe zu machen. Weiter stehen Freizeitaktivitäten zur Wahl, für die es allerdings oft Wartelisten gibt. Die Aktivitäten beinhalten Deutsch und Englisch als Fremdsprache ${ }^{1}$. Bildung senkt das Risiko der Rückfälligkeit, wie Stud-

1 Die Problematik der Fremdsprachigkeit ist im hiesigen Justizvollzug nicht neu und daher haben Deutschlernangebote für nicht muttersprachliche Gefangene eine historisch lange Tradition, es gab sie bereits Ende des 19. Jahrhunderts. Die Schulleitung und das Unterrichten übernahm der Pfarrer, Hilfslehrer und manchmal auch noch Mitgefangene unterstützen ihn. ln den beiden deutschsprachigen Konkordaten (die Schweiz hat insgesamt drei) bieten die Anstalten ja nach Grösse und finanziellen Ressourcen den Gefangenen Deutschunterricht für Fremdsprachige (DaF)an. Eine Übersicht über die Angebote wurde nie erstellt. 2007 trat das revidierte Strafgesetzbuch in Kraft. Der Artikel $75^{*}$ stellt die Bildung der Arbeit gleich. Durch die gesetzliche Verankerung wurde der Deutschunterricht der Häftlinge zur Bildungsmassnahme. 
ien gezeigt haben (The Center on Crime, Communities \& Culture: 2001), aber Deutsch im Besonderen ist auch deshalb wichtig, weil es dem Gefangenen bei der Integration und Resozialisierung hilft. Es erleichtert ausserdem den kommunikativen Alltag zwischen Gefangenem und Anstaltsmitarbeitendem. Zudem sind die Sprachkenntnisse im Deutschen oft eine Voraussetzung für eine Psychotherapie oder auch eine Ausbildung.

\subsection{Der Kurs}

Der Unterricht findet in einem Klassenzimmer statt, das, mit Whiteboard, Hellraumprojektor und Fernseher mit Video ausgestattet, sich kaum von einem anderen Schulzimmer für Erwachsenenbildung unterscheidet. Es sind sechs Lerngruppen von zwischen drei und zehn Schülern, die jeweils einmal pro Woche 50 Minuten Unterricht haben. Trotz der durchschnittlichen Haftdauer von drei Jahren ist die Anstalt alles andere als ein stabiler Ort und so verändern sich auch die Gruppen oft. Es kommt zu Verlegungen in andere Anstalten, Gefangene werden entlassen oder nach dem Ende ihrer Strafe des Landes verwiesen.

Wenn die Anzahl Lernende auf eine minimale Zahl zurückgegangen ist, rücken jeweils neue von der Warteliste nach. Ein Einstufungstest hilft den für jeden neuen Lernenden am besten geeigneten Platz in einer Gruppe zu finden. Das Einteilen ist oft ein schwieriges Unterfangen, einerseits sind natürlich die Deutschkenntnisse massgebend, aber genauso wichtig ist, ob ein Neuer in eine Gruppe passt und wie sich durch ihn die Gruppendynamik (vielleicht zum Negativen) verändern könnte. Des Weiteren sind Neuzugänge ab und zu eine Störquelle, da ein Einzelner seinen Platz in einer bestehenden Gruppe erst finden muss und von den „Alteingesessenen" nicht immer mit offenen Armen empfangen wird. Im Unterricht kann oder muss von heterogenen Gruppen ausgegangen werden. Normalerweise gibt es eine oder zwei Anfängergruppen/"falsche Anfänger" und weitere Gruppen die sich irgendwo zwischen A2 und $\mathrm{C} 1$ des Gemeinsamen europäischen Referenzrahmens (GER) bewegen.

\subsubsection{Prüfen und Testen}

Die Wiedereingliederung und die Integration sind die Hauptziele für Programme im Justizvollzug und wenn man sich die bisher genannten Besonderheiten dieses Lehr- und Lernkontextes vor Augen führt, so kann es nicht im Wesentlichen darum gehen, die Gefangenen auf eine Prüfung vorzubereiten. Nichtsdestotrotz haben aber die Gefangenen die Möglichkeit, sich auf ein oder auch mehrere Diplome des Goethe-Instituts auf ihrer Stufe vorzubereiten. Hierfür gibt es jedoch keine offiziellen Termine, was Druck von den Lernenden und der Lehrperson nimmt. Immer wenn es wieder Prüfungskandidaten gibt, wird ein Datum festgelegt und die Prüfungen auf den verschiedenen Stufen vorbereitet. Die Prüfungskandidaten können selbstverständlich über eine Teilnahme mitbestimmen, sie sollten auf jeden Fall gute Chancen haben, die Prüfung zu bestehen, sonst wird ihnen empfohlen bis zum nächsten Termin zu warten. Ein Scheitern bei den Prüfungen soll den Lernprozess eines Gefangenen nicht behindern. Dieser Ansatz führt ab und an zu schwierigen Entscheidungen: So war ein Lernender einmal sehr niedergeschlagen, als ihm die Lehrerin eine schlechte Prognose für einen Prüfungserfolg gab und ihm vorschlug erst noch weiterzulernen.

Trotz allem sind Prüfungen wichtig, denn sie geben der Lehrerin einen Anhaltspunkt, zeigen, was gelernt wurde, und sind Voraussetzung für Entscheidungen $\mathrm{zu}$ neuem Stoff. Diese Ansicht vertritt Rudman (1989), der Lernen und Lehren als Zusammenspiel sieht. Leider fehlt im Unterricht die Zeit, um das Gelernte neu aufbereitet $\mathrm{zu}$ wiederholen, zu recyceln, obwohl dies vom Unterrichtsmaterial her angeboten würde. Als Lehrende muss man da 'Mut zur Lücke' beweisen. Abschliessend bleibt zum Testen zu sagen, dass es dem Lernenden den persönlichen Fortschritt signalisiert und ihn motiviert weiterzugehen.

\subsection{Die Lernenden}

Die Lernenden sind normalerweise zwischen zwanzig und etwa fünfzig Jahren alt, wobei es tendenziell mehr ältere Schüler gibt. Sie kommen von überall auf der Welt. Über sie kann man nicht als Gruppe schreiben, das wäre unmöglich, denn ihre Persönlichkeitsprofile unterscheiden sich stark in Bildung und Beruf, Interessen und Lernerfahrungen.

\section{Ansichten/Überzeugungen:}

\subsection{Zu den Gefangenen als Sprachlernern}

Das Taxonomiemodell eines guten Sprachenlerners (Skehan 1989) bietet einen Rahmen mit Kategorien, die mit dem Lernprozess direkt verbunden sind. Dank dieses Rahmens lassen sich Unterschiede unter den Lernern festmachen: Alter, Intelligenz, Talent, Motivation, Einstellungen, Persönlichkeit und Kognition. Weiter variiert der kulturelle und soziale Hintergrund. Während vielleicht einige Lernende ein Studium begonnen oder abgeschlossen, eine Berufslehre gemacht haben, haben andere nicht einmal die obligatorische Schulzeit absolviert. Die Klassen sind damit äusserst 
heterogen. Wie kann unter solchen Vorzeichen überhaupt effektives Lehren bzw. Lernen stattfinden, mag man sich da fragen. Muss es bei einer solchen Heterogenität unter den Lernenden nicht zwangsläufig zu Aggressionen unter den Einzelnen und disziplinarischen Schwierigkeiten der Lehrperson kommen?

Obschon die Gefangenen alle ihre individuelle Geschichte haben, leben sie doch allesamt in einer streng durchorganisierten und strukturierten Welt mit klaren Abläufen. Der Kontext schafft also eine gewisse Homogenität. Die Situation im Freiheitsentzug ist für mehr oder weniger alle Gefangenen ,häufig per se belastend..." (Christoffel und Schönfeld 2008: 10). Diese Belastung kommt etwa durch verminderte Energie, eine deprimierte Grundhaltung und reduzierte Präsenz zum Ausdruck. Folglich werden unter den Deutschkursteilnehmern immer wieder ähnliche Muster im Lernverhalten zu finden sein: Fehlende Flexibilität und Spontanität und fehlendes Interesse. Und sicher dürfte es auch eine Reihe von Gefangenen geben, die sich durch den Deutschkurs eine Stunde von der Arbeit ausklinken können und für die dies die einzige Motivation ist.

Im Gegensatz zum oben Gesagten kommt man als Lehrende doch immer wieder zum Schluss, dass die Lernenden mit Motivation Deutsch lernen und dass sie bemüht sind Fortschritte zu machen. Aber der Spracherwerb ist kein linearer Prozess und viele der Lernenden müssen mit einem u-förmigen Verlauf klar kommen: Ihre Motivation ist zunächst sehr gross und sie sind davon überzeugt, dass die Fremdsprache in den Griff zu bekommen ist. Später finden sie diese „extrem schwierig", wie sich ein Lernender ausdrückte, und sie stolpern über verschiedene Wissenslücken: „Den deutschen Artikel werde ich nie beherrschen!" Um ihre Defizite zu überwinden, möchten sie gern mehr Kontaktstunden, was natürlich ausser Frage steht: „Ich werde dem Direktor einen Hausbrief schreiben. Der wäre auch nicht imstande eine Sprache mit einer Stunde Unterricht zu lernen. " Über ihr geringes Vorwärtskommen oder die Stagnation im Lernen sind sie häufig frustriert und manche geben an diesem Punkt das Deutsch lernen auf.

Auf der anderen Seite gibt es Lernende, die eine gewisse Sprachängstlichkeit entwickeln, die sich nicht von selbst wieder verflüchtigt, wie Oxford (1999) unterstreicht. Die beiden Situationen sind heikle Punkte während des Lernprozesses und müssen überwunden werden. Hier ist die Reaktion der Lehrperson entscheidenden, wie das folgende Beispiel darlegen soll:
Ein Lerner war begeistert von der Idee, ein Goethe-Diplom auf der Stufe B1 ablegen zu können. Obwohl er gewisse Defizite hatte, hielt die Lehrerin seinen Wunsch für realistisch. Da eine Unterrichtsstunde pro Woche für eine Prüfungsvorbereitung sehr knapp ist, sollte er in seiner Freizeit den Teil „Schreiben“ trainieren. Auf dieser Stufe war die Schreibaufgabe damals die Antwort auf einen halbformellen Brief, dessen Inhalt in Form von Notizen bereits vorhanden war. Der betroffene Lernende begann so:

„, Lieber Herr Lutz, ich habe überhaupt keine Übung im Schreiben eines Briefes.

Liebe Frau Schumacher, ich entschuldige mich dafür, dass ich so ein ignoranter Idiot bin. Ich habe gar keine Idee, wie ich auf diesen Brief antworten sollte. Ich bin mit dreizehn aus der Schule. Ich würde meine Situation sehr gern verbessern, mit Ihrer Hilfe könnte ich es schaffen. Ich bekomme das in den Griff. Vielen Dank für Ihr Verständnis. Freundliche Grüsse... “

Dieser Lernende verliess die Anstalt mit einem B1-Zertifikat und sein Brief bekam eine der besten Noten unter den Prüflingen auf dieser Stufe.

Oft scheinen verbesserte Fremdsprachenkompetenzen einen positiven Effekt zu haben, der auch auf den Alltag ausstrahlt. Zum einen wirkt sich ein Erfolgserlebnis natürlich positiv auf das Selbstwertgefühl aus: Ein Lernender hatte während des Unterrichts jeweils sein Deutschdiplom bei sich und blickt ab und zu etwas träumerisch drauf. Ein anderer Lernender berichtete, dass er dank der Alphabetisierung und seiner fremdsprachlichen Fortschritte nun in der Lage sei, ein Formular auszufüllen, und zwar ohne Hilfe. Wie Quinn (2007) sagt, sind solche und ähnliche Erfolge für die Lehrperson unschätzbar wertvoll.

Wie sehr sich Kursteilnehmer im Lernprozess in die Rolle als Lernende versetzen können, zeigt folgendes Beispiel:

Es ging um Nebensätze und die Lernenden sollten eigene Beispiele suchen. Ein Schüler sagte: „Da ich einen Migräneanfall habe, würde ich gern auf meine Zelle gehen." Er gab diesen Satz in etwa fünf Versionen wieder, alle waren sie korrekt und die Lehrerin lobte ihn. Als sie dann aber in seine Richtung schaute, rollte er seine Augen und seine Gesichtsfarbe changierte zwischen gelb und weiss. Er musste in einer sehr schlechten Verfassung sein, dennoch verwendete er die Strukturen absolut korrekt. 
Nachdem es bisher um individuelle Beispiele gegangen ist, steht im folgenden Teil die Gruppendynamik unter den Gefangenen im Zentrum: Einige Lernende wollen keine Hausaufgaben machen und sie wissen, dass dies für die Lehrerin in Ordnung ist, andere erwarten aber, dass sie bis zum nächsten Unterricht Vertiefungsmaterial bekommen und es macht ihnen Spass die Resultate untereinander zu vergleichen. Hier ein Beispiel aus dem Unterrichtsalltag, das diesen Punkt unterstreicht: ,,Sie, Frau S., mein Lernpartner hat sich beklagt, weil Sie uns das letzte Mal so wenig Aufgaben mitgegeben haben. Können wir bitte das nächste Mal mehr haben?"

\subsection{Zur Unterrichtsführung und Methodik}

„Ist das nicht gefährlich, dort zu unterrichten?" ist eine häufige Frage von Kolleginnen und Kollegen. Es ist klar, welche Ansichten hinter dieser Frage stehen: Eine Lehrperson in einem Gefängnis begibt sich in eine gefahrenreiche Umgebung und muss sowohl die Situation unter Kontrolle haben, aber auch die Lernenden. So könnte man zum Schluss kommen, dass hier Frontalunterricht, bei welchen die Lehrerin bzw. der Lehrer im Zentrum steht, angezeigt ist. Tatsächlich wird im Deutschunterricht der Anstalt so unterrichtet. Die Tische bilden dabei eine U-Form. Allerdings hat dieser Ansatz weniger mit Fragen der Sicherheit zu tun, vielmehr zeigte sich, dass die Lernenden es bevorzugen, den Anweisungen der Person, die sie unterrichtet, zu folgen, abwechslungsweise Fragen zu beantworten, die Aufmerksamkeit und das Interesse der Person vor ihnen zu bekommen und auf keinen Fall isoliert zu sein. So würden die Lernenden stets die Arbeit im Plenum der Einzelarbeit vorziehen; Letztere sei für die Zelle.

\subsection{Zum Einsatz und Sinn der Muttersprache im Unterricht}

Die Anstalt ist dezentral geführt, die Gefangenen leben in Zellen, die sich in Wohngruppen befinden. Um das Übergewicht einer bestimmten Ethnie auf einer Gruppe und mögliche, damit verbundene disziplinarische Probleme $\mathrm{zu}$ vermeiden, sind Landsleute meist in verschiedenen Pavillons untergebracht. In den Deutschgruppen kann es aber durchaus vorkommen, dass eine Ethnie übervertreten und somit vielleicht auch dominanter ist. Da die meisten Lernenden sich auf den Sprachniveaus A1 und A2 des Gemeinsamen europäischen Referenzrahmens bewegen, vertritt die Lehrerin die Ansicht, dass ein moderater Gebrauch der Muttersprache durchaus vertretbar und im Lernprozess sinnvoll ist. $\mathrm{Zu}$ diesem Schluss kommt auch die Zweitspracherwerbsforschung (SLA): Kellermann
z.B. (1986) zeigt, dass der Gebrauch der Erstsprache (L1) ein subtiler und sich entwickelnder Aspekt im Erwerb der Zweitsprache (L2) ist. Darüberhinaus kann die Verwendung der Erstsprache dazu führen, dass bei der lernenden Person ein echtes Interesse am Vergleich von Aspekten der beiden Sprachen entsteht und dass dabei Analogien ausgemacht werden. Wenn die Lernenden Phänomene ihrer eigenen Muttersprache auf Deutsch erklären, sind sie selber Lehrer und Dolmetscher auf Zeit. Da die Zielsprache Deutsch ist, kann der Lernende erkennen, ob er sich selber verständlich machen kann. So kann es sich positiv auf die sprachliche Leistung des Lernenden auswirken, wenn er eine Verbindung zwischen der Muttersprache und dem Deutschen macht, das zeigt Forschung, z.B. Heyde (1979). Ein schöner Nebeneffekt ist dabei, dass die Lehrperson ihren kulturellen Horizont erweitert und die eigenen Fremdsprachen trainiert.

Ein Lernender wusste z.B. nicht, was das Wort „Aprikose“ bedeutet. Seine Kollegen übersetzten es für ihn ins Arabische. Worauf er sagte: „Ah, mushamsh.“ - Das klang wie „Mischmasch“ für uns nicht Arabisch Sprechenden. Dies ist nur ein triviales Beispiel, das zeigt, wie ein Wort, ein Homophon das Interesse der Lernenden wecken kann und so zum kulturellen „Erlebnis" wird.

\subsection{Zu Ansätzen und Zugängen}

Die Bedürfnisse der Deutsch Lernenden im Gefängnis lassen sich grob in zwei Kategorien einteilen: Einmal geht es um das dringende Bedürfnis, zu kommunizieren und zu verstehen, bei der Arbeit, beim Arzt. Zum anderen soll die deutsche Sprache längerfristig die Resozialisation und die Wiedereingliederung in die Gesellschaft erleichtern. Und so läge es eigentlich auf der Hand, dass im Unterricht hauptsächlich die vier Fertigkeiten - Lesen, Hören, Schreiben und Sprechen trainiert werden müssten. Wenn man noch berücksichtigt, was weiter oben über die Lernenden gesagt wurde, dann erscheint ein Ansatz von der Grammatik her weniger angebracht.

Welche sprachlichen Kenntnisse und Fähigkeiten brauchen die Gefangenen dringend? Sie haben ja in ihrem Alltag und bei der Arbeit mit Anstaltsmitarbeitenden zu tun, weiter mit Anwälten, Therapeuten und Sozialarbeitern, sie müssten also vorrangig Kompetenzen erwerben, die ihnen die mündliche und schriftliche Kommunikation erleichtern. Die Grammatik lässt sich dabei eher vernachlässigen und man entscheidet sich für einen eher spielerischen Ansatz. Die Teilnehmen- 
den fühlen sich so wohl und können ihre oft schwierige Situation für den Moment vergessen. Jedoch, kamen einige Sprachspiele nicht sehr gut an bei den Klassen:

„Also wissen Sie, das Spiel, bei dem es zwei Spieler gibt und alle anderen Schiedsrichter sind, erinnert mich an die Situation vor Gericht. Der Richter thront hoch über mir. Ich möchte das nicht mehr spielen. “

Solche und ähnliche Aussagen von Lernenden machten die Lehrerin nachdenklich. Sie suchte nach neuen Wegen, gleichzeitig fragten Schüler immer wieder nach Parallelen und Unterschieden zwischen ihrer Muttersprache und der deutschen Sprache. Das war die Entscheidung für expliziten Grammatikunterricht. Nicht alle waren davon hellauf begeistert. Ein Lerner meinte:

„Einst sprach ich wie einer von der Baustelle - von der Grammatik her völlig falsch. Aber die Leute verstanden mich mehr oder weniger. Und jetzt kommen Sie mit ihrer Grammatik und nun arbeitet die Sprache in mir und ich muss mir immer wieder Dinge überlegen. "

Diese Äusserung kam verärgert daher und selbstverständlich war der betroffene Lernende mit dem neuen Ansatz nicht einverstanden. Aber im Bezug auf die Theorie des Zweitspracherwerbs stand fest, dass es hier um die Sensibilisierung für grammatische Phänomene ging. Diese Idee der Bewusstmachung geht auf Rutherford und Sharwood Smith (1988: 107) zurück: Instruktion bringe nicht automatisch Produktion hervor, und so müsse versucht werden, die Aufmerksamkeit der Lernenden gezielt auf die formalen Eigenschaften der Zielsprache zu lenken. Die Lehrende wählt einen induktiven Ansatz des Grammatikunterrichts. Den Lernenden hilft es, wenn ihnen bewusst wird, dass die deutsche Sprache - die ,unglaublich schwierig“ ist - fünf grammatische Fälle hat, während ihre Muttersprache über sieben verfügt. Sie bewegen sich innerhalb der Fremdsprache sicherer und selbstbewusster, denn die Grammatik zeigt ihnen auf, wie die „Mechan$\mathrm{ik}^{\text {" }}$ der Sprache funktioniert.

\subsection{Zu Themen}

Anfänglich fiel es der Leherin schwer, sich für Themen für den Unterricht zu entscheiden. Persönlich war sie eher der Ansicht, dass die Gefangenen im Unterricht nicht noch zusätzlich mit ihrer schwierigen Situation konfrontiert werden sollten. Es erschien zunächst klar, dass das Thema „Kriminalität und Strafe“ im Kursbuch auf der Stufe B2/C1 ausgelassen werden müsste. Beim Durchgehen der Themen im Inhaltsverzeichnis wurde der Lehrerin aber bewusst, dass jegliches ,allgemeine Thema" schnell den sicheren Boden verlassen und die Gefangenen in einer nicht intendierten Weise blossstellen könnte. Es waren dann allerdings die Gefangenen selber, die ihr zeigten, dass ihre Bedenken unbegründet waren.

Nachdem die Lehrerin gerade erst ein paar wenige Wochen in der Anstalt unterrichtet hatte, brachte ein Gefangener einen Cartoon mit. Die Hauptfigur war ein kleiner Vogel auf seinem Motorrad. Einmal hat der Vogel einen Unfall, in den ausser ihm auf der Maschine noch ein Auto verwickelt ist. Der Vogel verliert das Bewusstsein. Als er wieder zu sich kommt, befindet er sich in einem Käfig. Er sieht den Wasserspender und ein paar Scheiben Brot auf dem Boden und glaubt, er sei im Gefängnis: „, Oh nein, ich muss den Autofahrer getötet haben. " Der Gefangene schaute die Lehrerin an und kicherte, sie war ziemlich geschockt.

Ein anders Mal ging es um eine Stelle im Kursbuch der Stufe A1: Da gab es einen Dialog zwischen einem kleinen Mädchen und einem Pizzaservice-Mitarbeitenden. Das Mädchen telefoniert mit dem Service, um neun Pizzas zu bestellen. Weil sie minderjährig ist, will die Person am Ende der Leitung die Mutter oder den Vater sprechen. Doch das Mädchen ist mit dem Hund allein zu Hause. Ihre Bestellung wird mit den Worten: „, Tja Lisa, keine Mama, kein Papa - keine Pizza. “ abgeschmettert. Die Lernenden kommentierten darauf: „Dieses arme Mädchen ist in genau derselben Situation wie wir."

In vielen Situationen sind es die Gefangenen selber, die ihre eigene aktuelle Situation zur Sprache bringen, wie die folgenden Beispiele zeigen: Wenn die Lernenden nach Komposita gefragt werden, bringen sie meistens Begriffe wie „Fluchtgefahr“ oder „Haftstrafe“. Einmal fragte ein Gefangener: „Ist das korrekt, wenn ich sage, mir gefällt es hier?" Die Lehrerin antwortete, dass es aus grammatikalischer Sicht richtig sei, die Mitgefangenen lachten.

Manchmal wird das Thema „Kriminalität“ auf ganz natürliche Art und Weise ins Spiel gebracht. Ein Gefangener bereitete sich sehr ernsthaft auf ein Diplom der Stufe B1 vor. Er war auf einem Ohr leicht schwerhörig und er wollte speziell den Prüfungsteil „Hören“ gut vorbereiten. So bat er die Lehrerin um Prüfungsbeispiele, die er in der Zelle hören konnte. Er bekam das entsprechende Material, wurde aber darauf aufmerksam gemacht, dass er die CDs in einer Woche 
wieder intakt zurückbringen müsse. Er meinte darauf: Keine Sorge,: „, Ich schliesse meine Zelle immer sorgfältig ab, wenn ich zum Beispiel zum Duschen weggehe. "Vielleicht fixierte die Lehrerin ihn dann einen Moment zu lange, denn er fügte hinzu: „Oh, ich verstehe, nein, ich werde die nicht verkaufen oder so. "

Ein anderes Mal meinte ein Gefangener zur Lehrerin, dass ihm gar nicht aufgefallen sei, dass sie Linkshänderin sei: „,Ich wusste nicht, dass Sie Linkshänderin sind. Machen Sie alles mit der linken Hand? Schreiben Sie mit ihr? Brauchen Sie sie bei manueller Arbeit? Sorry, aber mit welcher Hand schiessen Sie? - Ich habe eine Pistole gebraucht. Was, Sie haben keine Waffe? Ich dachte, alle Schweizer hätten eine. “

\section{Fazit}

Vielleicht inspiriert dieser Artikel Lehrende zum Nachdenken - über eigene Ansichten und Überzeugungen was das Unterrichten generell, aber besonders auch im Gefängniskontext begrifft. Die Autorin des Artikels kann aus eigener Erfahrung sagen, dass der besondere Unterrichtskontext im Vollzug ihre Ansichten und Überzeugungen immer wieder auf die Probe stellt. Für die Erfahrungen, die sie sammelt, ist sie sehr dankbar.

\section{References}

Christoffel, Ueli/Schönfeld, Frank. 2008. Es gilt die Zeit zu nutzen. Deliktpräventive Psychotherapie mit Straftätern. Psychoscope. S. 8-11. Vol. 29. 10/2008. Bern: Föderation der Schweizer Psychologinnen und Psychologen FSP.

Goethe Institute München. [online], abrufbar unter: <URL: http://www.goethe.de/>

Hadley, C. 1943 John Cotton Dana: A Sketch. Chicago: American Library Association.

Hattie, John. 2009 Visible learning. Routledge, London, New York.

Heyde, A. 1979 The relationship between self-esteem and the oral production of a second language. Unpu -blished doctoral dissertation, University of Michigan.

Kellermann, E. 1986 Crosslinguistic constraints on the development of the L2 lexicon. In Kellermann, E. \&
Sharwood Smith, M. (eds.), Crosslinguistic Influence in Second Language Acquisition. New York: Pergamon, 35-48.

McDonough, J. 1994 A teacher looks at teachers' diaries. ELTJ Vol 48/1, 57-65.

Oxford, R.L. 1999 Anxiety and the language learner: new insights. In Arnold, J. (ed.), Affect in language learning. Cambridge: Cambridge University Press.

Pajares, M.F. 1992 Teachers' Beliefs and Educational Research: Cleaning up a Messy Construct. Review of Educational Research, Vol. 62, No.3; 307-332.

Poeschwies. [online], abrufbar unter: <URL: http://www.Justizvollzug.zh.ch/content/justizvollzug/ poeschwies/index.html>.

Puchta, H. 1999 Creating a learning culture to which students want to belong: the application on Neuro-Linguistic Programming to language teaching. In Arnold, J. Affect in language teaching. Cambridge: Cambridge University Press.

Quinn, C. 2007 Teaching in a prison: a reflection. In Fieldnotes for ABLE STAFF 2007 Edition. [online], abrufbar unter:

<URL: http://www.able.state.pa.us/fieldnotes07>.

Rudman, H.C. 1989 Integrating testing with teaching. Practical Assessment, Research and Evaluation [online], 1(6). abrufbar unter: $<\mathrm{URL}$ : http://www.ericae.net/pare/getvp?=1\&n=6.>.

Rutherford, W. and Sharwood-Smith, M. (eds.) 1988 Grammar and second language teaching: a book of readings. New York: Newbury House.

Skehan, P. 1989 Individual differences in second-language learning. London: Edward Arnold.

Sinclair, J. (ed.) 1995 Collins Cobuild English Dictionary. London: Harper Collins Publisher.

The center on crime, communities \& culture, op. Cit., 5.

Willis, J. 1996 A framework for task-based learning. Harlow: Longman.

Yero, J.L. 2001/2002 Beliefs. Teacher's mind resources. [online], abrufbar unter: <URL: http://teachers mind.com $>$.

Monika Schumacher ist Dozentin für Deutsch als Fremdsprache an der Fachhochschule Zürich. Sie unterrichtet auch Deutsch an der Hochsicherheitsgefängnis "Pöschwies" (Zürich). Sie stützte ihre Doktorarbeit in der Soziolinguistik an der Kommunikation zwischen inhaftierten Nicht-Muttersprachler der deutschen und der Wärter in einer Justizvollzugsanstalt. 